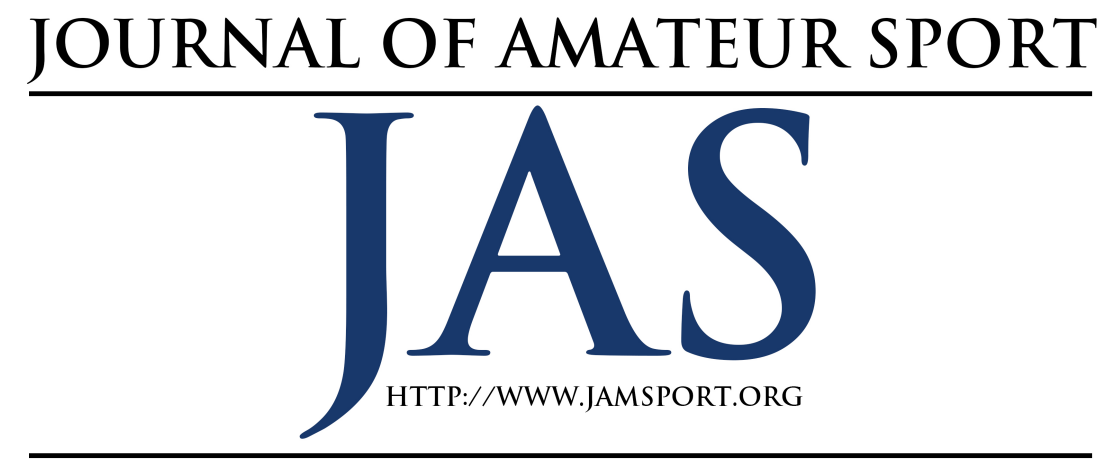

\title{
Enhancing the Transfer of Life Skills from Sport-based Youth Development Programs to School, Family, and Community Settings
}

\author{
Jennifer M. Jacobs ${ }^{1}$ \\ Michael Lawson ${ }^{2}$ \\ Victoria Nicole Ivy ${ }^{2}$ \\ Kevin R. Richards ${ }^{2}$ \\ ${ }^{1}$ Northern Illinois University \\ ${ }^{2}$ The University of Alabama
}

\begin{abstract}
Sport-based youth development programs represent a promising approach for engaging youth in activities that can support their socioemotional, physical, and life skill development. This article focuses on the strengths, opportunities, and challenges related to the design and implementation of an established sport-based activity model called Teaching Personal and Social Responsibility (TPSR). This TPSR approach revolves around activities that are designed to help youth develop life skills that can extend to multiple areas of their lives, including the family, community, and school. Notwithstanding this important strength, our review of the literature suggests that this transfer process may be far from automatic, especially for low-income youth challenged by poverty and its correlates. To address this difficulty, we offer an expanded social-ecological framework. This framework highlights opportunities for program leaders, researchers, and evaluators to better ground TPSR programs in surrounding community systems and in youth's emergent cultural strengths and world views.
\end{abstract}

S port-based youth development programs (SBYD) have received increased educational practice and policy attention in recent years, and for good reason. When designed appropriately, these physical activity programs provide youth with multiple social, educational, and life benefits (Holt, Neely, Slater, Camiré, Côté, Fraser-Thomas, MacDonald, Strachan, \& Tamminen, 2017). These benefits derive from opportunities for enhanced social connections to peers, 
teachers, coaches, and other caring adults at school and in the community (Hemphill \& Richards, 2016; Martinek \& Lee, 2012).

They then extend to include other important indicators of school and life success such as grit, self-regulation, and selfdetermination (Gould \& Carson, 2008; Petitpas, Cornelius, Van Raalte, \& Jones, 2005).

One of the more popular SBYD programs to emerge in recent years is the Teaching Personal and Social Responsibility (TPSR) model (Hellison, 2011). This TPSR model is gaining popularity because of its unique focus on helping youth develop a set of life skills and related competencies that they can apply to multiple areas of their lives, including school.

Although the TPSR literature asserts the model's potential for helping youth develop and transfer these skills and competencies, our review of the broader educational and SBYD research literature suggests that this transfer process may be far from automatic, especially for youth from underserved communities (Lee \& Martinek, 2013). For instance, while the process of life skills and competency transfer may initially depend on students' skill development, youth may not complete the transfer process if their teachers, peers, and family members do not support the direct use and application of content learned in the program (Lave, 1997). For this reason, the overall success of SBYD programs and their transfer related goals may ultimately depend on the extent to which the norms, practices, and priorities of schools, families, and SBYD programs are properly synchronized and harmonized (i.e. their social ecology). (Martinek \& Lee, 2012).

The purpose of this paper is to provide an expanded social-ecological lens and framework for understanding how the transfer-related goals of TPSR and related SBYD programs might be better aligned, integrated, and synchronized with their surrounding school, family, and community systems. In pursuit of this purpose, we begin with a brief overview of the TPSR model, and a TPSR program that is currently being implemented and studied in a low-income community in the southeastern region of the United States. This program offers several "real world" case examples that help illustrate the importance of attending to the social ecology of sport-based youth development. Next, we highlight opportunities to better connect family, school, and neighborhood practices and norms in support of positive youth development. One particular model for school-community practice is offered as an exemplar for this work; an emergent approach to parent involvement and family support called Collective Parent Engagement (Alameda-Lawson \& Lawson, 2016). We conclude the article with select implications for future research and development on TPSR and related SBYD programs. 


\section{Teaching Personal and Social Responsibility}

As indicated in its name, the TPSR model aims to support the development of two fundamental competencies in youth: (a) personal responsibility; and (b) social responsibility. Personal responsibility refers to youth efforts to exercise individual skills such as respect, effort, and self-control. Social responsibility is defined as being aware of other's rights and needs and responding to them in pro-social, and culturally desirable ways. The model was developed with a set of goals that help to focus lesson content and work toward the achievement of each of these goals (Hellison, 2011). The first four TPSR goals include (a) respect and self-control, (b) participation and showing effort, (c) selfdirection, and (d) caring about others and leadership. The fifth goal involves the application or transfer of the first four goals outside of the gym in other areas of youths' lives (Martinek \& Lee, 2012).

Each TPSR session begins with relationship time, where program leaders interact with youth to develop personal relationships and trust. Following relationship time, program leaders give an awareness talk where the TPSR focus of the day is introduced, based on a selected goal (e.g., respect). During this discussion, program leaders provide youth the opportunity to describe what they believe the focus means, typically by inviting them to give examples of what it looks or sounds like (e.g., what does it look like when someone shows respect?) (Hellison, 2011).

The next and most time-intensive segment of each TPSR session is the lesson focus. During this time, sport or physical activity lessons are delivered in a way that integrates opportunities for youth to observe, practice, and apply life skills. While some of these opportunities may be structured into the plan for the lesson (e.g., having students practice the goal of helping others by asking more skilled students to help less skilled students with a task), program leaders can also identify "teachable moments" related to the goal to prompt youth to consider and discuss ways the goal of the day could be better achieved. Teachable moments occur when an educator responds to inquiry on the spot, when the learner is ready to learn new information (Rich, 2009). For example, if children are expected to practice dribbling a ball with their feet, but they kick it across the gym instead, there is an opportunity to teach them about self-control.

Following the lesson focus, TPSR instruction involves youth in discussions that are designed to enhance their overall understanding of the session goal. Here, the instructor might ask youth to identify examples of their peers effectively demonstrating the goal for the day, or asking them to discuss how interpersonal conflicts were addressed throughout the lesson.

The final lesson segment is reflection time. Here, youth are asked first to evaluate their 
performance related to the day's goal. Then, they are asked to consider how the activities and goal of the day might be used to enhance the behavior at home, school, and other social settings (Hellison, 2011).

\section{The Out-of-School Time Program}

Our appreciation of the strengths, opportunities, and challenges facing TPSR and related SBYD programs is derived, in large part, from our own work as designers, facilitators, and evaluators of similar programmatic efforts. In this section of the article, we describe the emergent challenges associated with the development and implementation of TPSR as they have presented themselves in a particular, out-ofschool-time program (OST).

The school-community targeted by this OST effort is a low-income, predominately African-American community that is challenged by high rates of unemployment as well as on-going social exclusion dynamics, following a long history of racial segregation that remains largely unresolved today. In 2011, the community was essentially decimated by a tornado, which destroyed most of the housing and foliage in the area, as well as the elementary school targeted by the effort. The school re-opened two years ago and now serves nearly 450 students, $87 \%$ of which qualify for government-sponsored, free or reduced lunch, indicating the family comes from a low socioeconomic status. Academically, the school ranks below the 30 th percentile in student test scores in the state (Lawson, Alameda-Lawson, \& Richards, 2016).

Below we provide an overview of the program, and then refer back to it in examples provided throughout the remainder of the discussion. The examples have been collected as part of an ongoing qualitative research initiative that currently spans one year and includes ethnographic observations and interview data from youth participants and pre-service physical education teachers.

The OST program is led principally by a university faculty member, doctoral student, and preservice physical education teachers, and meets afterschool three days a week in the gym of the collaborating elementary school. The program serves approximately seventy youth in grades 1-5 who are referred for participation by the school personnel (i.e., teachers, principals, counselors, and social workers) based on being at risk for social, behavior, and academic issues. The faculty member and doctoral student ran the sessions for 6-8 weeks at the beginning of each semester. During that time, the preservice teachers were led through lectures and peer-teaching sessions for six hours a week as training for participation in the program. These training sessions were created around the guidelines of the Children Moving (Graham, Holt, Hale, \& Parker, 2013) and the Teaching Personal and Social Responsibility Through Physical Activity Model (Hellison, 2011) textbooks to develop the pre-service teachers' pedagogical and model-based knowledge and skills. From 
here, pairs of pre-service teachers began to develop their own lesson plans and miniunits with consistent feedback from their professor and taught them to small groups during the OST program twice a week, with the third day still led by the faculty member and graduate student.

Utilizing TPSR guidelines, youth in the OST program are taught fundamental movement skills (e.g., hopping, skipping, throwing and catching, kicking) and movement concepts through the skill themes approach to elementary physical education (Graham, Holt, Hale, \& Parker, 2013), in addition to a daily focus on developing personal and social responsibility. Each session is run in a similar fashion, in line with the TPSR lesson format (Hellison, 2011). First, youth informally interact with each other and the leaders for several minutes during relationship time at the start of the session. Program leaders then introduce the TPSR goal of the day (e.g., participation/effort, respect) during an awareness talk, and prompt youth to describe what they believe the goal means, looks like, sounds like, etc.

Next program leaders conduct a physical activity lesson introducing participants to movement concepts and skills that vary from popular sports to nontraditional activities, such as folk dancing. Several strategies are used by the leaders to enhance the TPSR experience including giving choices and voices, assigning leadership roles, and promoting transfer (Escartí, Wright, Pascual, \&
Gutiérrez, 2015). For example, several times throughout the lesson, program leaders select instances to recognize children who are demonstrating the TPSR goal or provide feedback on how it can be demonstrated more effectively in sport, and likewise applied to life. Youth are frequently encouraged to make their own responsible choices without prompts from the leaders, while also meeting preset behavioral expectations (Hellison, 2011).

At the conclusion of the session, program leaders facilitate a group discussion related to the daily goal and youth are encouraged to rate how well they achieved the daily goal and share examples from the lesson that support their rating. Time is also reserved for individual reflection on life skill and physical activity learning prior to dismissal. A key component of both group discussion and individual reflection includes program leaders prompting youth to consider on how the TPSR goal can be applied outside of the program setting. Central to this program and all TPSR programs is the idea of transfer, or encouraging youth to seek opportunities to demonstrate positive behaviors in alternate settings such as their schools, homes, and community (Hellison, 2011).

\section{Transfer and Context (In)congruence}

As noted earlier, TPSR programs such as the OST program introduced above have the potential to teach children life skills that can influence multiple aspects of their lives (Hellison, 2011). This prospect of life skills 
and competency transfer is most likely when youths' surrounding school, family, and community contexts share similar norms and values (Lee \& Martinek 2013; Martinek, Schilling, \& Johnson, 2001). This process is typically referred to as "near transfer," with the adjective "near" indicating the contextlevel correspondence that makes skills and competency transfer easier or more manageable to youth (Gordon \& Doyle, 2015).

In contrast, when the cultural values and/or norms for acceptable behavior are inconsistent across social contexts, "far transfer" occurs (Gordon \& Doyle, 2015). "Far transfer" refers to instances in which children receive contradictory and confusing messages from the adults or peers in their lives about norms for acceptable behavior. Research indicates that these contradictory messages can undermine children's social and emotional learning (Melendéz \& Martinek, 2015). They can also put youth in the undesirable position of having to choose between conflicting allegiances of school, family, friends etc. (Fordham \& Ogbu, 1986).

We mention these challenges and tensions here because they have been consistently present in the OST program. For instance, the OST program sought to improve behavior by empowering youth to make choices and teach them how to exercise their voices appropriately, whereas authority figures from the school took a more direct approach to behavior management that emphasized obedience and submission to authority. Likewise, in the case of verbal or physical conflicts among students in school, teachers would frequently separate or isolate the involved parties rather than facilitating positive communication and eventual conflict resolution as the OST program promoted.

Along with school and program inconsistencies, individuals from children's families and kinship networks sometimes possess priorities and values that don't align with the philosophy of SBYD programs. These networks have critical implications for life skills transfer because family members are the primary socializing agents for their children (Clausen, 1966). For this reason, parents' involvement in SBYD programs often represents a determining factor in program success (Holt et al., 2017).

Meléndez and Martinek (2015) witnessed a lack of congruence between family and program norms and orientations toward the notion of respect. Specifically, they found that some of their program youth residing in underserved urban communities have been instructed by their families/kinship networks to gain "respect" from others by exerting power and influence over them. In contrast, in the TPSR setting, "respect" is typically defined as valuing the rights and feelings of others (Hellison, 2011). These kinds of disconnects position youth between the two forces of socialization, both of which they need to be healthy and successful. When youth lack the power, knowledge, or skills to reconcile these disconnects and contradictory 
messages, they are confronted with an affective dilemma (Phelan, Davidson, \& Cao, 1991).

The youth in the OST program have, at times, displayed patterns of violence when working through problems that manifest in the program. Following the TPSR model, leaders attempt to walk youth through problem solving strategies in the face of conflict such as taking a break from the situation, talking it out in a safe and structured way, and finding a common ground to compromise (Hellison, 2011). These efforts do not always translate into sustained positive behavior change because many youth in the OST program strongly believe that physical altercations solve problems. This is likely due to a lack of congruence between different contexts in their lives. For example, a fifth grade participant felt threatened by another boy during a game of basketball and hit him in the face. When leaders discussed the incident and attempted to lead the young man to consider other choices he may have made, he insisted that his mom told him to hit anyone whom he feels has acted in a threatening manner. He said we could call his mother to talk with her about the incident, but that would not matter because she would think that he did the right thing.

Another common theme with children in the OST program is the way they comprehend and respond to authoritative figures. For example, when the children are prompted to talk about respect, they define it as, listening to the adult who is talking.
When asked why they specify adults instead of just listening to others, youth explained that they are told their job is to be respectful by being quiet and listening to adults at home and in school. Though this rationalization, youth demonstrate a behaviorist response rather than an autonomous, empowerment-based internalization of the value, as TPSR aims to foster. In the OST program setting, leaders promote the importance of mutual and earned respect for all, which conflicts with the ideology many youth have been taught about respect being conditional. Taken altogether, these kinds of dilemmas highlight needs for strategies that can enhance correspondence, or congruence, between school, family, and program contexts.

\section{Theoretical Perspective toward Congruence}

The preceding discussion has established that TPSR afterschool programs do not operate in isolation, but are instead embedded within larger school, family, and community contexts. All of these contexts interact, and incongruences (i.e., inconsistencies) and contradictions between them can negatively impact youth development (Banks \& Banks, 2004; Martinek \& Lee, 2012). To address this potential for harm, we turn to the collective parent model (Alameda-Lawson \& Lawson, 2016), which provides theoretical insight into how and why programs that integrate the TPSR framework might forge stronger 
connections with school and family contexts.

\section{Collective Parental Engagement}

Collective parent engagement (CPE) is a moniker used to describe programmatic efforts that engage parent groups in activities designed to improve school and community contexts (Alameda-Lawson, Lawson, \& Lawson, 2010). This collectivist approach differs from the conventional "Parent Involvement" (PI) approach highlighted in much extant educational research (e.g. Epstein, Sanders, Simon, Salinas, Jansorn, \& Voorhis, 2002). For instance, conventional PI programs are typically developed with the assumption that poor educational and social outcomes stem from low levels of parent involvement in home and school activities. Following these assumptions, PI efforts are typically designed to help "educate" or "inform" low-income parents about how they might engage in activities that can support the educational and social welfare of their children and school.

In contrast to this conventional view, $\mathrm{CPE}$ programs are designed with the assumption that the primary barriers to school success lie not in parents, but in larger structural issues and problems like poverty, social isolation and exclusion dynamics, crime, and community safety; and that low-income parents, families, and communities may already be doing as much as they can-as individuals - to support their children's health, welfare, and overall school success. As such, CPE efforts are typically designed to develop and mobilize social networks that can help parents address institutional-level problems that cannot be easily understood or changed by individuals working in isolation from one another (Ishimaru, Barajas-López, \& Bang, 2015; Lawson \& Alameda-Lawson, 2012).

Alameda-Lawson \& Lawson (2016) offer specific strategies through one $\mathrm{CPE}$ approach that includes three on-going, interactive, and iterative strands of programmatic activity (see also AlamedaLawson, Lawson, \& Lawson, 2013). The first phase, the individual parent engagement phase, includes the following activities: (a) door-to-door outreach to the homes of community parents; (b) a collaborative decision-making and needs assessment process that allows parents to individually and collectively identify barriers to their children's healthy development and overall social welfare; and (c) a 40-hour Outreach Training course that trains parents in community outreach, communication, family assessment and family interviewing, and agency referral.

Drawing from the strengths-based and engagement-focused skills emphasized in the outreach training course, the second design phase of their approach is developed to connect parents to others in the CPE program and neighborhood community (Alameda-Lawson et al, 2013). In this "collective development" phase, participating parents are provided with a small weekly stipend that supports their efforts to design and implement 
programmatic solutions to the barriers and challenges they identified during the assessment/individual engagement phase. For example, the stipend could be used for a Home Visitation program that enables parents to take lead roles in solving public health issues like a school-wide Lice outbreak or chronic truancy problems at school (Alameda-Lawson et al., 2013).

The third phase of CPE centers on the development of a school-community collaborative. This collaborative is developed to enhance family access to, and use of, school-community services and resources. When effectively implemented, past versions of these collaboratives have facilitated significant shifts/improvements in the design and delivery of schoolcommunity services (Alameda-Lawson \& Lawson, 2016; Lawson \& Alameda-Lawson, 2012). One example of this shift is the transfer of professional roles from solo service providers to a more flexible, bottom-up approach that builds on family and community strengths. Research indicates that when professionals learn to work with parents and leverage their strengths, more sustainable "anchoring" support contexts are created in the community (e.g. Netter Center for Community Partnerships, 2008; Warren, Nelson, \& Burlingame, 2009). These anchoring supports facilitate the development of social capital and collective efficacy in the community while enhancing "horizontal" linkages between schools, health and human services, and youth development agencies (Alameda-Lawson \& Lawson, 2016; Lawson \& Alameda-Lawson, 2012).

\section{Enhancing cultural adaptivity.}

Because CPE targets change in school, neighborhood, and community-contexts, it represents a logical partner for TPSR and other SBYD programs. One way that CPE can support the transfer-related goals of TPSR and SBYD programs is by helping school parents and children learn how to adapt to institutional cultures, practices, and norms that are different from their own. For example, the school-community consortium of families, educators, and community services developed by CPE provides families and school-community professionals with a joint opportunity to develop shared norms for family, school, and community interaction. Sport programs should look for opportunities to be involved in this process to foster greater alignment with different contexts and in turn provide youth with targeted strategies for navigating across contexts and developing cultural adaptivity. The development of these shared norms provides an opportunity to address the kinds of context incongruences that might otherwise limit the skills and competency transfer goals of TPSR and SBYD programs.

The CPE model can help foster this cultural adaptivity in several ways. For instance, during the effort's outreach training course, parents learn about the behavioral norms of school and 
governmental service systems and are taught communication skills that enable them to negotiate the barriers often experienced by low-income parents and families in these settings. Second, CPE provides opportunities for routine interactions between parents and school/neighborhood service contexts. Among other potential benefits, these interactions provide families with the necessary opportunity to learn how to negotiate different cultural norms and contexts, often with the support of a trained family advocate/social worker (Lawson \& Alameda-Lawson, 2012).

Finally, CPE provides consistent opportunities for children to see their parents engaged at the school and in other community/institutional settings, often in tandem with the adults (e.g., teachers, coaches, social workers) who are charged with their day-to-day care and well-being. Theoretically, these kinds of interactions help to reduce the feelings of affective dissonance/ambivalence that low-income children and youth may experience when their families and schools work in isolation, or even worse, in competition with one another (e.g., Lawson, 2003). All in all, the theoretical and empirical benefits associated with CPE provide an important rationale for wedding TPSR programs with efforts that can help reduce cultural disconnects and divides between families, schools, and neighborhood contexts.

\section{Enhancing the Transferability of Lessons Learned in TPSR Programs}

Parents and families play a critical developmental role in their children's lives, including the realm of physical activity and sport (Dorsch, Smith, \& McDonough, 2009; Dorsch, Smith, Wilson, \& McDonough, 2015). Through this paper we attempt to illustrate that a collective understanding of the youth participants, coaches, teachers, and institutional structures (e.g., schools, community organizations) is necessary to facilitate the positive outcomes youth experience from sport, exercise, and physical activity, and to help them transfer lessons learned to other areas in their lives. Based on an understanding that youths' environments are complex and comprised of multiple contexts that at different times conflict, align, interact, and coexist, there is a need to explore how TPSR programs can be better aligned with surrounding socialinstitutional systems.

In Figure 1 we present a conceptual framework that begins with the assumption that students are best able to transfer lessons learned through TPSR programs to other contexts in their lives when the goals and values across those contexts are more similar than dissimilar. Within this paper, we specifically discuss the program, family and school contexts as these are all experienced by youth in the program, but as indicated in the model, "other social contexts" (e.g., peers, religion, non-profit organizations, etc.) also interact and provide a considerable influence over youth development as well. 
This model illustrates that TPSR program providers should work toward empowering youth to help them recognize, navigate, and adapt to differences across these contexts, while also working to bring those contexts toward congruence.

The circle around the outside of the figure represents the community context in which all contexts operate. The family, school, TPSR program contexts are then depicted on points of a triangle representing the need to build connection and congruence, with the mediating factor of other social contexts. The arrows on the outside of the triangle reflect the influence that each context exerts on the others. Specifically, there is interplay between the family, school, program, and other contexts, and each context socializes youth to behave in specific ways (Lee \& Martinek, 2013). One goal of TPSR program providers is to work with school leaders and parents to bring the three contexts closer together to better facilitate transfer inter-context transfer. Another goal is to develop adaptivity in youth, which feeds back into how they approach the family and school contexts. Opportunities for transfer outside of the program are enhanced when students perceive congruence across contexts and when they develop adaptivity in navigating these contexts.

\section{Strategies for Developing Congruence across Contexts}

The unifying message of our approach to better facilitating transfer from TPSR- focused afterschool programs relates to building congruence across social contexts. We provide recommendations related to recognizing barriers to congruence; and coalition-building between TPSR programs and other social contexts.

\section{Recognizing barriers to congruence.}

The first strategy we propose for developing congruence across contexts involves programs recognizing youth as products of multiple worlds, and that goals espoused in these contexts sometimes conflict. It is imperative that programs identify the potential for incongruent values at home and school before proposing strategies for students to mediate these differences. Because incongruences are context and site specific, program leaders need to develop relationships and get to know youth participants, their schools, and their community/family situations. This aligns with the SBYD best practice of relationship building (Holt et al., 2017), and positions program leaders to think about students' lives in a holistic way so they can be better equipped to teach youth the skills they need to navigate across contexts.

In addition to adopting a more holistic viewpoint of youths' lives, another strategic approach is for programs to promote certain values that give youth the opportunity to think about how their different life contexts relate and overlap. Inherent to the TPSR model is the value of youth empowerment, or recognizing and affirming students' strengths (Hellison, 2011). One way this can be leveraged to foster congruence is by 
encouraging youth to recognize their families' strengths as a part of their own, and having program leaders respond in ways that show respect for student and family situations (Bryan \& Henry, 2008). Youth, particularly those from marginalized backgrounds, bring an awareness of their families' struggles into the program setting. This is an opportunity for program leaders to support their dialogue and help youth see how their families have demonstrated qualities of resilience and perseverance.

Social responsibility, one of the pillars of TPSR, is another value that provides the opportunity for youth to make connections across family, school, community, and program life. Research has suggested that teaching social responsibility is considered a primary function of schools, almost ranked with an equal emphasis to academic skills (Liem, 2016). Because teachers value order and responsible behavior in the classroom, TPSR programs are in a unique position to advocate for the value of the program as aligning with and promoting school behavioral initiatives. When students receive social responsibility lessons in their sport programs that encourage respecting the rights and feelings of others and cooperation, and these values are further fostered in the classroom, students receive congruent messages that further support adopting this behavior. Program leaders, then, have the potential to impact classroom culture and even school culture to where school personnel could see the program as a highly supportive entity to their values and goals (Blair \& Raver, 2015; Cadima, Verschueren, \& Buyse, 2015).

\section{Coalition-building between the} program and other social contexts.

With established values in the program setting that encourage program leaders to view youth in a more holistic manner, programs can turn their attention to coalition building, or fostering relationships between the program, families, and schools (Benson, 2003 Martinek \& Lee, 2012). Consistent with CPE (Lawson \& AlamedaLawson, 2012), it is necessary to establish an authentic dialogue between program providers, school personnel, and families where all voices play a unique developmental role in youths' lives (Debnam, Johnson, Waasdorp, \& Bradshaw, 2014).

First, building alignment and a shared sense of initiatives between programs and parents is a critical part of fostering congruence. Because TPSR programs are typically situated within school contexts, programs often inherit the challenges that derive from parent-school partnerships as well (Hellison, 2011). Among the many barriers parents experience related to school engagement, research has explored isolation as one significant factor (Delgado-Gaitan, 1991; Greenwood \& Hickman, 1991). Namely, to parents, programs associated with the school can be perceived as foreign or exclusionary since parents of marginalized students were often not afforded the same opportunities in their educational experiences (Turney \& Kao, 
2009). Thus, one factor that leads to parental disengagement from school-related events is the powerlessness they feel in influencing school culture. Several tactics can serve to overcome this challenge. For example, outreach events situated in the sport setting where parents and peers are invited and can experience the program norms are encouraged. This could include hosting a parent sport night where youth present the values and goals of the program and then participate in a culminating sporting contest or open gym session open to members of their families. Other options include creating an awards ceremony where all youth receive an honorary participation award for completing the program with their parents in attendance.

As an added benefit, building relationships in the sport setting between parents and youth at a time when they are more engaged in their child's lives (e.g., elementary school) increases the likelihood that they will remain engaged in their sport experiences in the long term (Wuerth, Lee, \& Alfermann, 2004). Furthermore, when parents are given access to the program setting, they not only observe the core values of the program in action, they witness their child interacting with peers, which forges a connection between youths' home and social lives (Phelan et al., 1991). Providing parents the regular opportunity to watch the program and attend regular events further contributes to building and sustaining alignment between youths' contexts.
Getting parents to attend school events can be challenging, particularly when youth come from families where both parents work or when other life demands take precedence over school events (Goodall \& Montgomery, 2014). Thus, programs can encourage youth to share the program values with parents and exemplify them at home. In many ways, quality programming that promotes enjoyment makes youth naturally want to share their experiences with parents, teachers, and peers. However, programs can further foster a connection between the program and family setting through assigning "transfer" homework, such as asking youth to "perform one leadership act at home," or "show respect to a classmate who is having a difficult day." This can be further supported through sending information home to parents about what youth are learning in the program setting (Hellison, 2011), as supporting research has demonstrated that parents want schools and teachers to advise them about how to best help their children at home by keeping them apprised of what their children are doing at school (Epstein \& Dauber, 1991).

Finally, TPSR programs should consider the role program leaders have outside of formal program hours. Research has examined the positive impacts that can result from schoolteachers interacting with parents at the end of the day (RimmKaufman, \& Pianta, 1999). This same basis can apply to TPSR program leaders interacting with parents after the program 
ends. Making program leaders available and approachable to parents creates a more positive and open environment.

Additionally, forging relationships between program staff and parents contributes to the TPSR mission as program leaders increase their impact and solidify themselves as caring adult figures that have compassion for youth outside of the program.

Programs also have the potential to foster congruence between the school setting and the program by creating an opportunity for youth to exhibit what they learn through the program at school. Schools struggle with a lack of capacity and available resources to holistically focus on students and barriers to learning and development, particularly in underfunded, urban environments (Bryan \& Henry, 2008). However, since students' ability to move between their different "worlds" greatly affects their school success, TPSR programs are in a position to provide institutional structures that help support students' investment and engagement in school (Phelan et al., 1991). Programs could, for instance, encourage school personnel to provide opportunities for meaningful student participation based on the lessons they learn in the program (Bryan \& Henry, 2008). This could include forming a peer mediation team or conflict resolution plan, based off the sport program content (e.g., learning about self-control and problem solving), as a way to remedy school behavioral issues.

Some TPSR research has recognized the school counselor or school social worker as a good point of access for this type of intervention (Gordon, Jacobs, \& Wright, 2016). Program leaders should look for opportunities to build mutually beneficial and sustainable partnerships with school personnel who are invested in students' developmental success and privy to the non-academic struggles that students face (Jacobs, Condon, \& Wright, 2014; Cook, Hayden, Bryan, \& Belford, 2016). For example, program staff could invite the school counselor/social worker's involvement by referring students to join the program, or even attending sessions and participating in the physical activity/sport lessons to gain an understanding on the goals of the program. Further discussions with these key school stakeholders could center on adopting a shared language or values system related to TPSR, or keeping an open dialogue about managing student behaviors in a way that moves from militaristic approaches into more empowerment-centered frameworks.

One approach that has been developed primarily within sport-based community programs is the concept of growing the programs' reach in the greater community context (Jacobs, Castaneda, \& Castaneda, 2016). The majority of SBYD and TPSR work has been situated within controlled physical spaces where the nature of the program setting provides limited access for outsiders (e.g., gyms, classrooms, church buildings, community centers). While these are opportune launching pads for initially establishing positive relationships, teaching 
values, and encouraging healthy behaviors, we recommend that that program leaders should increase the visibility of the program reach so that youth have the opportunity to exercise their life skill development outside of the controlled program setting. Notably, outside environments such as unsafe neighborhoods, unstable home situations, and failing schools pose a great risk to youth being able to sustain developmental gains (Buckle \& Walsh, 2013; Lerner, Agans, Arbeit, Chase, Weiner, Schmid, \& Warren, 2013). Thus, community-based sport programs have focused their efforts on empowering youth to act in positive ways in these same public spaces through initiatives such as organizing public sporting events and service/outreach activities (AndersonButcher, Iachini, Ball, Barke, \& Martin, 2016; Jacobs et al., 2016). It is through these engaged community experiences, under the program direction, that youth can build the confidence to apply their life skills to new, unfamiliar, and oftentimes quite different school, community, and cultural contexts.

While the research in this area with respect to sport programs fulfilling this role is still emerging, one model communitybased sport program situated in the inner city of Chicago has seen success through enacting a variety of different public events aimed at creating acceptable public spaces and changing the culture of norms present in a historically gang-controlled area (Jacobs et al., 2016). For instance, this organization promotes the use of "drop-in" programs as a solution for appealing to those youth who are not committed to regularly attending a program, but may establish a pattern of attendance if they are less pressured to fully commit. These are often youth who are labeled as "on the fringes," or vulnerable for risk factors such as drug use, gang involvement, or risky sexual behaviors (Sandford, Armour, \& Warmington, 2006). A drop-in option enables youth to participate in sports while also providing them with important access to a safe, inclusive environment that is sponsored by caring and supportive staff. This format also allows regular program attendees to bridge the gap between their social lives and the program with the option to invite friends to participate.

Other community-based program components include hosting sports tournaments in outdoor spaces that community residents can attend as spectators or volunteers. These events subscribe to a "strength in numbers" approach in an attempt to change youths' perceptions of living in a community that is dominated by gangs, and instead demonstrate how neighborhoods can be "taken back" by the presence of groupings of positive allies (Jarrett, 1997). Creating programs where parents and community members can become connected with the program and volunteer as coaches or referees creates further opportunities for involvement in youth sport experiences (Cuskelly, 2004).

\section{Other Opportunities for}

Development and Research. Finally, there 
are several measures that TPSR program leaders can take to help youth develop the kinds of individual and collective competencies fostered by CPE. For example, TPSR program leaders can help youth learn about the concepts and then help them reflect about how norms differ depending on setting and context. These kinds of learning and reflection activities can be conducted during the "relationship time," "activity," and "reflection" periods of each TPSR lesson.

To provide a more concrete illustration, a TPSR teacher could start a relationship talk by asking kids about how they define "respect" and then encourage/support a certain definitional view of respect during the skills-development portion of the lesson. Next, during the reflection period, the teacher could encourage youth to explore and share how the notion of "respect" varies according to different contexts, settings, and social environments. Subsequent lessons could then be devoted to helping youth learn how to effectively adapt to, and reconcile, competing norms and definitions of the same concept. By helping youth learn how to reconcile the contradictory norms and identity conflicts posed by competing settings, program leaders (with proper training focused on developing cultural competencies) can help TPSR programs become key mediating settings for positive youth development (see also Lawson \& Alameda-Lawson, 2012). Such is the potential of youth development programs that are anchored in a strong, social-ecological framework.

\section{Conclusions and Final Thoughts} Programs that implement the principles of SBYD represent a promising strategy for helping low-income children and youth develop the kinds of life skills, competencies, behaviors, and social identities they need for educational and life success. Among these SBYD approaches, the TPSR model has emerged as a special exemplar and leading best practice model (Holt et al., 2017). The TPSR model has earned this "best practice" status not only because of its multi-pronged focus on youth empowerment, relationship building, and life skills development, but also because of its unique and explicit strategy of helping children and youth learn to transfer competencies to other school, family, and community contexts.

Although we assert the promise and potential of TPSR and SBYD to improve child and youth outcomes, our analysis indicated that in some cases their current program design may not be sufficient for helping youth transfer the skills they learn in program settings to other contexts and community contexts. To this point, the research literature is clear that skills and competency transfer is best facilitated when children's school, family, and community environments are governed by similar values and expectations for desired behavior (Gordon \& Doyle, 2015). Unfortunately, the literature indicates that, in many low-income 
communities, this "correspondence" or "congruence" in desired norms and social practices does not exist across school, family, and community contexts. Consequently, these disconnects loom as significant structural barriers to the successful design and implementation of programs using the TPSR framework.

Several important conclusions can be derived from this important finding and claim. The first is that, in order to help get the conditions right for skills and competency transfer, program leaders should strive to marry TPSR/SBYD programs with broader efforts, like CPE, that can help them and other community leaders better synchronize and harmonize school, family, and community resources in support of the youth's optimal development. But to make this important leap, TPSR/SBYD will need to expand its orientation from a stand-alone program (often in afterschool settings) to a socialecological intervention that targets organizational and context development (e.g., Unger, 2011). Although the conceptual underpinnings of this expanded, collective work was sketched in this article (i.e., Figure 1), this context integration oriented work stands as the next frontier.

The second conclusion that can be derived from this paper pertains to those communities where harmonizing and synchronizing norms between family, school, and community is either not desired or logistically feasible. In such instances, we encourage TPSR program leaders to adopt a more explicit focus on helping program children and youth learn how to transfer the skills, talents, and identities they develop in the program to settings that have different, and at times, competing norms and values. But to pursue this important work, TPSR program leaders will need to make youth adaptability across contexts an explicit, targeted learning competency, and pursuing this important goal may require the development of expanded program curricula as well as additional staff training.

The third and related conclusion relates to the professional preparation that is needed to enhance SBYD programs that serve low-income, youth, families, schools, and communities. Currently, youth service providers are trained in a variety of academic disciplines and helping fields. Notwithstanding the potential strengths of this diversity, the sheer range and scope of the current education and training pipeline threatens to create a field that is not multidisciplinary as intended, but atheoretical and adisciplinary. To this point, our analysis indicates that future TPSR teachers and programs leaders will need explicit education and training in several academic fields and disciplines, and this may require the development of new interdisciplinary degrees and professional preparation programs. In our view, these new academic and professional preparation programs will include a robust focus on physical education and coaching, educational leadership, educational psychology (particularly theories on motivation, engagement, and transfer), 
social-ecological theory, critical and cultural theory, and organizational development. Moreover, given the inherent interdisciplinary nature of the work that lies ahead, inter-professional training and practice-embedded professional learning opportunities should be prioritized by the academic units/departments that are charged with developing the nextgeneration workforce for positive youth development programs.

Of course, all of these recommendations and the research from which they are derived stand as possibilities for the important work that lies ahead. To the extent this paper helps to shine the light on the future directions and potential for SBYD programs that serve youth, families, and communities, it will have achieved its primary purpose. 


\section{References}

Alameda-Lawson, T., \& Lawson, M. A. (2016). Ecologies of collective parent engagement in urban education. Urban Education, 1-36. doi.org/10.1177/0042085916636654 Alameda-Lawson, T., Lawson, M. A., \& Lawson, H. A. (2013). An innovative collective parent engagement model for families and neighborhoods in arrival cities. Journal of Family Strengths, 13(1), 1-25.

Alameda-Lawson, T., Lawson, M. A., \&

Lawson, H. A. (2010). Social workers' roles in facilitating the collective involvement of low-income, culturally diverse parents in an elementary school. Children \& Schools, 32(3), 172-182.

Anderson-Butcher, D., Iachini, A. L., Ball, A., Barke, S., \& Martin, L. D. (2016). A University-School Partnership to Examine the Adoption and Implementation of the Ohio Community Collaboration Model in One Urban School District: A MixedMethod Case Study. Journal of Education for Students Placed at Risk (JESPAR), 21(3), 190-204. doi.org/10.1080/10824669.2016.118 3429

Benson, P. L. (2003). Developmental assets and asset-building community: Conceptual and empirical foundations. In R. M. Lerner \& P. L. Benson (Eds.), Developmental assets and asset-building communities: Implications for research, policy, and practice (pp. 19-43). Norwell, MA: Kluwer Academic. Blair, C., \& Raver, C. C. (2015). School readiness and self-regulation: $\mathrm{A}$ developmental psychobiological approach. Annual Review of Psychology, 66(1), 711-731.

Bryan, J., \& Henry, L. (2008). Strengthsbased partnerships: A school-familycommunity partnership approach to empowering students. Professional School Counseling, 12(2), 149-156. doi.org/10.5330/PSC.n.2010-12.149

Buckle, M. E., \& Walsh, D. S. (2013). Teaching Responsibility to GangAffiliated Youths. Journal of Physical Education, Recreation \& Dance, 84(2), 53-58.

Cadima, J., Doumen, S., Verschueren, K., \& Buyse, E. (2015). Child engagement in the transition to school: Contributions of self-regulation, teacher-child relationships and classroom climate. Early Childhood Research Quarterly, 32(1), 1-12.

Clausen, J. A. (1966). Family structure, socialization, and personality. In L. W. Hoffman \& M. L. Hoffman (Eds.), Review of Child Development Research (Vol. 2, pp. 1-53). New York, NY: Russell Sage Foundation.

Cook, A. L., Hayden, L. A., Bryan, J., \& Belford, P. (2016). Implementation of a School-Family-Community Partnership Model to Promote Latina Youth Development: Reflections on the Process and Lessons Learned. The 
International Journal of Research on

Service-Learning and Community

Engagement, 4(1).

Cuskelly, G. (2004). Volunteer retention in community sport organisations. European Sport Management Quarterly, 4(2), 59-76.

doi.org/10.1080/1618474040873746 9

Debnam, K. J., Johnson, S. L., Waasdorp, T. E., \& Bradshaw, C. P. (2014). Equity, connection, and engagement in the school context to promote positive youth development. Journal of Research on Adolescence, 24(3), 447-459.

Delgado-Gaitan, C. (1991). Involving parents in the schools: A process of empowerment. American Journal of Education, 100(1), 20-46.

Dorsch, T. E., Smith, A., \& McDonough, M. H. (2009). Parents' perceptions of child-to-parent socialization in organized youth sport. Journal of Sport and Exercise Psychology, 31, 444-468. doi:

http://dx.doi.org/10.1123/jsep.31.4. 444

Dorsch, T. E., Smith, A., Wilson, S. R., \& McDonough, M. H. (2015). Parent goals and verbal sideline behavior in organized youth sport. Sport, Exercise and Performance Psychology, 4, 19-35. doi.org/10.1037/spy0000025

Epstein, J. L., \& Dauber, S. L. (1991). School programs and teacher practices of parent involvement in inner-city elementary and middle schools. The Elementary School Journal, 91(3), 289-305.

Epstein, J. L., Sanders, M. G., Simon, B. S., Salinas, K. C., Jansorn, N. R., \& Van Voorhis, F. L. (2002). School, family, and community partnerships: Your handbook for action. Corwin Press.

Escartí, A., Wright, P. M., Pascual, C., \& Gutiérrez, M. (2015). Tool for Assessing Responsibility-based Education (TARE) 2.0: Instrument revisions, inter-rater reliability, and correlations between observed teaching strategies and student behaviors. Universal Journal of Psychology, 3(1), 55-63.

Fordham, S., \& Ogbu, J. U. (1986). Black students' school success: Coping with the "burden of 'acting white'. The Urban Review, 18(3), 176-206.

Goodall, J., \& Montgomery, C. (2014). Parental involvement to parental engagement: a continuum. Educational Review, 66(4), 399-410.

Gordon, B., \& Doyle, S. (2015). Teaching personal and social responsibility and transfer of learning: Opportunities and challenges for teachers and coaches. Journal of Teaching in Physical Education, 34(1), 152-161. doi.org/10.1123/jtpe.2013-0184 Gordon, B., Jacobs, J. M., \& Wright, P. M. (2016). Social and emotional learning through a teaching personal and social responsibility-based after school program for disengaged middle school boys. Journal of Teaching 
in Physical Education. 35(4), 358-369.

doi.org/10.1123/jtpe.2016-0106

Gould, D., \& Carson, S. (2008). Life skills development through sport: Current status and future directions.

International Review of Sport and Exercise Psychology, 1(1), 58-78.

doi.org/10.1080/1750984070183457 3

Graham, G. Holt, N., Hale, S.A., \& Parker, M. (2013). Children moving: A reflective approach to teaching physical education (9th ed.). New York, NY: McGraw-Hill.

Greenwood, G. E., \& Hickman, C. W. (1991). Research and practice in parent involvement: Implications for teacher education. The Elementary School Journal, 91 (3), 279-288.

Hellison, D. (2011). Teaching personal and social responsibility through physical activity (3rd ed.). Champaign, IL: Human Kinetics.

Hemphill, M. A., \& Richards, K. A. R. (2016). Without the academic part, it wouldn't be squash: Youth development in an urban squash program. Journal of Teaching in Physical Education, 35(1), 263-276. doi.org/10.1123/jtpe.2015-0109

Holt, N. L., Neely, K. C., Slater, L. G., Camiré, M., Côté, J., Fraser-Thomas, J., \& Tamminen, K. A. (2017). A grounded theory of positive youth development through sport based on results from a qualitative meta-study. International Review of Sport and Exercise
Psychology, 10, 1-49.

doi.org/10.1080/1750984X.2016.118 0704

Ishimaru, A. M., Barajas-López, F., \& Bang, M. (2015). Centering family knowledge to develop children's empowered mathematics identities. Journal of Family Diversity in Education, 1(4), 1-21.

Jacobs, J. M., Castañeda, A., \& Castañeda, R. (2016). Sport-based youth and community development: Beyond the Ball in Chicago. Journal of Physical Education, Recreation \& Dance, 87(5), 18-22.

doi.org/10.1080/07303084.2016.115 7386

Jacobs, J.M., Condon, B., \& Wright, P.M. (2014). Project Leadership: Creating a partnership for serving marginalized youth through an afterschool sport program. Professional Development Schools Partners 10(1), 15-11.

Jarrett, R. L. (1997). Bringing families back in: Neighborhood effects on child development. Neighborhood Poverty, 2(1), 48-64.

Lawson, M. A., \& Alameda-Lawson, T. (2012). A case study of school-linked, collective parent engagement. American Educational Research Journal, 49(4), 651-684.

Lave, J. (1997). The culture of acquisition and the practice of understanding. Situated cognition: Social, semiotic, and psychological perspectives, In Kirshner, D., \& Whitson, J. A. (Eds.). Situated 
cognition: Social, semiotic, and psychological perspectives. (pp. 63-82). Psychology Press.

Lawson, M. A. (2003). School-family relations in context parent and teacher perceptions of parent involvement. Urban Education, 38(1), 77-133.

Lawson, M. A., Alameda-Lawson, T., \& Richards, K. A. R. (2016). A university-assisted, place-based model for enhancing students' peer, family, and community ecologies. Education Sciences, 6(2), 16-30.

Lee, O., \& Martinek, T. (2013).

Understanding the transfer of valuesbased youth sport program goals from a bioecological perspective. Quest, 65(1), 300-312. doi.org/10.1080/00336297.2013.791 871

Lerner, R. M., Agans, J. P., Arbeit, M. R., Chase, P. A., Weiner, M. B., Schmid, K. L., \& Warren, A. E. A. (2013). Resilience and positive youth development: A relational developmental systems model. In Handbook of resilience in children (pp. 293-308). Springer: US.

Liem, G. A. D. (2016). Academic and social achievement goals: Their additive, interactive, and specialized effects on school functioning. British Journal of Educational Psychology, 86(1), 37-56. DOI: $10.1111 /$ bjep.12085 Martinek, T., Schilling, T., \& Johnson, D. (2001). Transferring personal and social responsibility of underserved youth to the classroom. The Urban Review, 33(1), 29-45. DOI: 10.1023/A:1010332812171

Martinek, T., \& Lee, O. (2012). From community gyms to classrooms: A framework for values-transfer in schools. Journal of Physical Education, Recreation and Dance, 83(1), 33-38; 51. doi.org/10.1080/07303084.2012.105 98709

Meléndez, A., \& Martinek, T. (2015). Life after Project Effort: Applying values acquired in a responsibility-based physical activity program. Revista Internacional de Ciencias del Deporte, 10(41), 258-280. doi.org/10.5232/ricyde2015.04105

Netter Center for Community Partnerships. (2008). Annual Report 2007-08. Engage, Empower, Educate: A Focus on University- Assisted Community Schools and Education Reform. Retrieved January 10, 2017, from http://www.upenn.edu/ccp/Netter. pdfl.

Petitpas, A. J., Cornelius, A. E., Van Raalte, J. L., \& Jones, T. (2005). A framework for planning youth sport programs that foster psychosocial development. Quest, 19(1), 63-80. doi: http://dx.doi.org/10.1123/tsp.19.1.6 3

Phelan, P., Davidson, A. L., \& Cao, H. T. (1991). Students' multiple worlds: Negotiating the boundaries of family, peer, and school cultures. Anthropology 
\& Education Quarterly, 22(3), 224-250.

DOI:

10.1525/aeq.1991.22.3.05x1051k

Rich, V. J. (2009). Clinical instructors' and athletic training students' perceptions of teachable moments in an athletic training clinical education setting. Journal of Athletic Training, 44(3), 294303.

Rimm-Kaufman, S. E., \& Pianta, R. C. (1999). Patterns of family-school contact in preschool and kindergarten. School Psychology Review, 28(3), 426-438.

Sandford, R. A., Armour, K. M., \&

Warmington, P. C. (2006). Re-

engaging disaffected youth through physical activity programmes. British Educational Research Journal, 32(2), 251271.

doi.org/10.1080/0141192060056916 4

Turney, K., \& Kao, G. (2009). Barriers to school involvement: Are immigrant parents disadvantaged? The Journal of Educational Research, 102(4), 257-271.

Ungar, M. (2011). Community resilience for youth and families: Facilitative physical and social capital in contexts of adversity. Children and Youth Services Review, 33(9), 1742-1748.

Warren, J. S., Nelson, P. L., \& Burlingame, G. M. (2009). Identifying youth at risk for treatment failure in outpatient community mental health services. Journal of Child and Family Studies,
18(6), 690-701. DOI:

10.1007/s10826-009-9275-9

Wuerth, S., Lee, M. J., \& Alfermann, D. (2004). Parental involvement and athletes' career in youth sport. Psychology of Sport and Exercise, 5(1), 2133. doi.org/10.1016/S14690292(02)00047-X 


\section{Figures}

Figure 1

Conceptual Model for Enhancing Skills and Competency Transfer Across Life Contexts. Solid arrow represent communication and congruence among the school, family, and TPSR program systems. Broken arrows reflect youth's ability to apply skills learned in SBYD program to different (and potentially competing) school, family, and community contexts.

\section{Conceptual Model for Enhancing Skills and Competency Transfer Across Life Contexts}

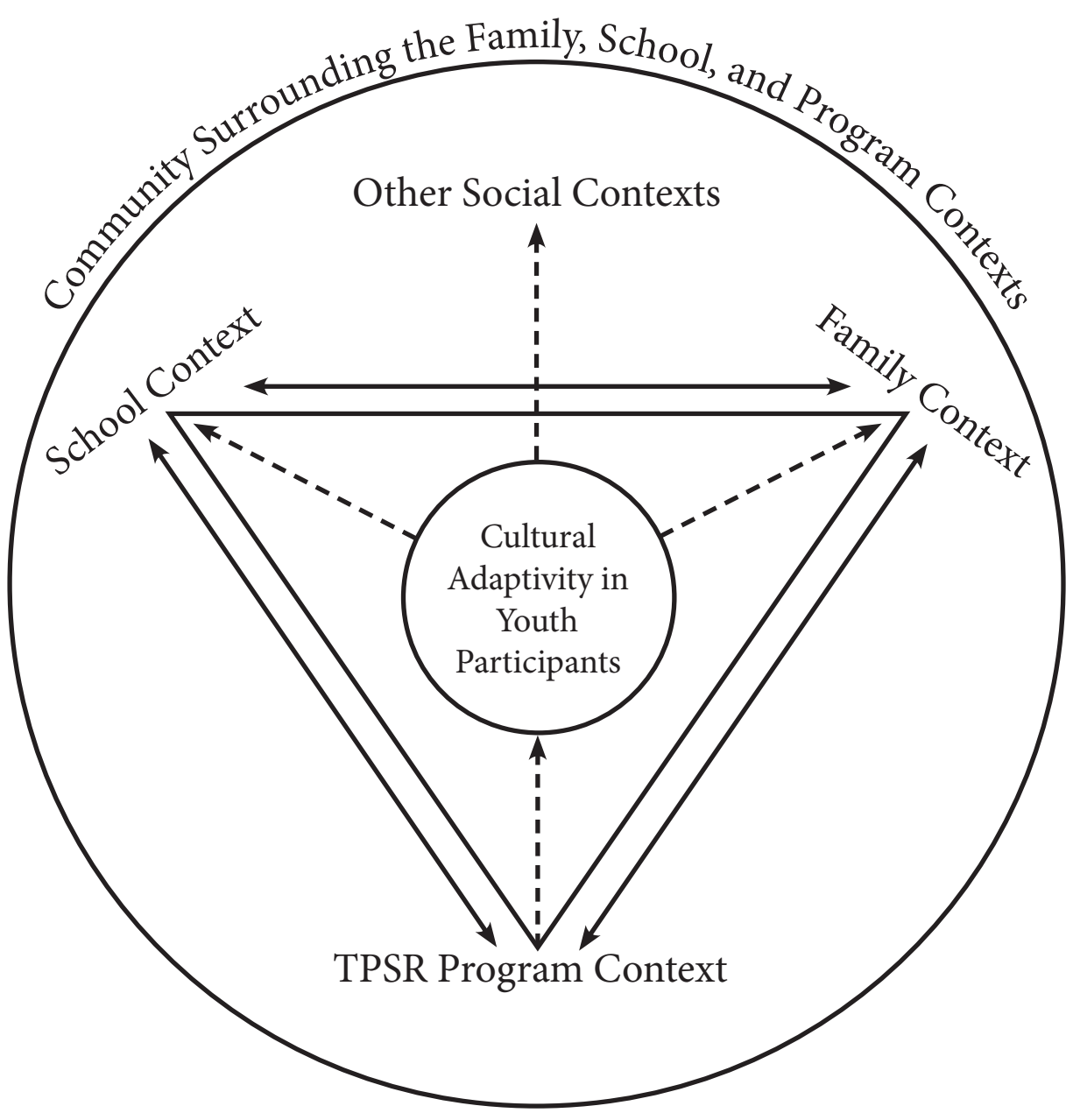

